EESTI NSV TEADUSTE AKADEEMIA TOIMETISED. IX KÖIDE FOOSIKALIS-MATEMAATILISTE JA TEHNILISTE TEADUSTE SEERIA. 1960, NR. 2

ИЗВЕСТИЯ АКАДЕМИИ НАУК ЭСТОНСКОИ ССР. ТОМ ІХ СЕРИЯ ФИЗИКО-МАТЕМАТИЧЕСКИХ И ТЕХНИЧЕСКИХ НАУК. 1960, № 2

\title{
О МЕТОДЕ НАИСКОРЕЙШЕГО СПУСКА ДЛЯ РЕШЕНИЯ НЕЛИНЕЙЫХ УРАВНЕНИЙ
}

\section{Л. Кивистик}

1. Пусть $P(x)$ есть нелинейный оператор из вещественного гильбертова пространства $H$ в то же пространство. Рассмотрим уравнение

$$
P(x)=0
$$

Ю. Лумисте [1] показал, что применение метода наискорейшего спуска для приближенного решения уравнения (1), если $P(x)$ потенциален (о потенциальности оператора см. [5]), равносильно нахождению последовательных приближений по рекуррентной формуле

$$
x_{n+1}=x_{n}+\varepsilon_{n} P\left(x_{n}\right), \quad n=0,1,2, \ldots
$$

где $x_{0}$ - начальное приближение, а $\varepsilon_{n}$ - вещественное решение уравнения

$$
\left(P\left(x_{n}\right), \quad P\left(x_{n}+\varepsilon P\left(x_{n}\right)\right)\right)=0 \text { * }
$$

Ю. Лумисте доказал сходимость метода в предположении, что опеpatop $P(x)$ удовлетворяет на некотором замкнутом множестве $X \subset H$ условию

$$
(P(x+h)-P(x), h) \geqslant m\|h\|^{2}, \quad m \geqslant 1
$$

Гуань Чжао-чжи $\left.{ }^{2}\right]$ доказал сходимость метода для дифференцируемого оператора в предположении, что для всех $x \in X$ выполнено условие

$$
\left(P^{\prime}(x) h, h\right) \geqslant m\|h\|^{2}, \quad m>0, \quad h \in H
$$

В этом же предположении, только с абсолютной величиной в левой части неравенства (5), некоторую модификацию метода наискорейшего спуска рассмотрел и $М$. Альтман $\left[{ }^{3}\right]^{* \star}$. Эту модификацию мы рассмотрим ниже, в пп. 4-6.

Практическая проверка условий (4) и (5) для всех $x$ целой области $X$ часто связана с большими трудностями. Ниже мы докажем сходимость метода наискорейшего спуска в предположении, что условие (5) с абсолютной величиной в левой части выполнено лишь для $x_{0}$.

- Символом $(u, v)$ обозначено скалярное произведение элементоз $u, v$ э $H$.

-. Отметим, что в [ $\left.{ }^{3}\right]$ условие $5^{\circ}$ теоремы 2 не выполнено, так как $B^{2}\left(E^{2}+D K\right)>$ $\geqslant 1+\frac{\|P(x)\|\left\|P^{\prime \prime}(x)\right\|}{\left\|P^{\prime}(x)\right\|^{2}} \geqslant 1$ и не может быть меньше единицы, как требуется в теореме 2. По той же причине не выполнены и условия теоремы 4 . 
2. Пусть оператор $P(x)$ дважды дифференцируем в смысле Фреше в некоторой замкнутой сфере $S\left(x_{0}, r\right)$, которую мы уточняем каждый раз, когда о ней идет речь. Потенциальности оператора мы не требуем. Пусть дифференциал $P^{\prime}(x) h$ удовлетворяет условию

$$
\left|\left(P^{\prime}\left(x_{0}\right) h, h\right)\right| \geqslant \frac{1}{M_{0}}\|h\|^{2}, \quad M_{0}>0
$$

для всех *夫 $h \in H$, где $x_{0}-$ начальное приближение решения уравнения (1). Последовательные приближения находим из рекуррентной формулы (2), где $\varepsilon_{n}-$ наименьшее по абсолютной величине решение уравнения (3). Но если $\varepsilon_{n}$ - решение этого уравнения, то (3) превращается в тождество

или

$$
\left(P\left(x_{n}\right), \quad P\left(x_{n}+\varepsilon_{n} P\left(x_{n}\right)\right)\right)=0
$$

$$
\left(P\left(x_{n}\right), \quad P\left(x_{n+1}\right)\right)=0
$$

Все следующие рассуждения остаются в силе, если уравнение (3) при $n=0,1,2, \ldots$ решено приближенно, но с такой точностью, что мы можем (учитывая точность нахождения $x_{n+1}$ ) считать

$$
\left(P\left(x_{n}\right), \quad P\left(x_{n+1}\right)\right)=0, \quad n=0,1,2, \ldots
$$

Т е о р м а 1. Пусть выполнены условия:

$1^{\circ}\left\|P\left(x_{0}\right)\right\|=\delta_{0} \leqslant \bar{\delta}_{0} \quad\left(x_{0}-\right.$ начальное приближение $)$;

$2^{\circ}\left|\left(P^{\prime}\left(x_{0}\right) h, h\right)\right| \geqslant \frac{1}{M_{0}}\|h\|^{2}, \quad M_{0}>0, \quad h \in H ;$

$3^{\circ}$ для всех $x \in S\left(x_{0}, r\right)$, где $r=\frac{1}{B}\left(\frac{1}{M_{0}}-\frac{1}{M^{*}}\right) \frac{\delta_{0}}{\bar{\delta}_{0}}$, оператор $P(x)$ дважды дифференцируем $и$

$$
\left\|P^{\prime}(x)\right\| \leqslant A, \quad\left\|P^{\prime \prime}(x)\right\| \leqslant B
$$

$4^{\circ}$ величины $M_{0}, \bar{\delta}_{0}, A$ и В такие, что последовательности $\left\{M_{n}\right\} \quad u$ $\left\{\bar{\delta}_{n}\right\}$, вычисленные из рекуррентных соотношений

$$
\begin{gathered}
M_{n+1}=\frac{M_{n}}{\sqrt{1-2 M_{n}^{2} B \bar{\delta}_{n}}}, \quad n=0,1,2, \ldots \\
\bar{\delta}_{n+1}=\bar{\delta}_{n} \sqrt{\frac{4 M_{n}^{2} A^{2}}{\left(1+\sqrt{1-2 M_{n}^{2} B \bar{\delta}_{n}}\right)^{2}}-1}, \quad n=0,1,2, \ldots
\end{gathered}
$$

сходятся.

Тогда уравнение (1) имеет в сфере $S\left(x_{0}, r\right)$ решение $x^{*}, \kappa$ которому сходится полученная из (2) последовательность $\left\{x_{n}\right\}, u$ справедлива оценка

$$
\left\|x^{*}-x_{n}\right\| \leqslant M^{*} \delta_{n} \leqslant \frac{\sqrt{2}}{A} \delta_{n}
$$

где $M^{*}=\lim M_{n} \quad u \quad \delta_{n}=\left\|P\left(x_{n}\right)\right\|$.

$$
n \rightarrow \infty
$$

- В дальнейшем, говоря о сфере $S\left(x_{0}, r\right)$, считаем ее замкнутой.

* Можно потребовать, чтобы условие (6) было выполнено только при всех $h$. для которых $\|h\|=\varrho$, где $\varrho-$ пронзвольное фиксированное положительное число. Но если условие выполнено для такнх $h$, то оно выполнено во всем пространстве. 
Доказ ательств о. Из (8) и требования сходимости следует, что $\left\{M_{n}\right\}$ есть возрастающая последовательность, а $\lim M_{n}^{2} B \bar{\delta}_{n}=0$. Поэтому $\left\{\bar{\delta}_{n}\right\}$ должна (монотонно) убывать. Теперь из (9) вытекает, что $\lim M_{n} A \leqslant \sqrt{2}$, или $M^{*} \leqslant \frac{\sqrt{2}}{A}$.

Обозначим

$$
f_{n}(\varepsilon)=\left(P\left(x_{n}\right), P\left(x_{n}+\varepsilon P\left(x_{n}\right)\right)\right)
$$

Тогда в силу условий теоремы

$$
\begin{aligned}
& \text { 1) } \frac{1}{\left|f_{0}^{\prime}(0)\right|}=\frac{1}{\left|\left(P\left(x_{0}\right), \quad P^{\prime}\left(x_{0}\right) P\left(x_{0}\right)\right)\right|} \leqslant M_{0} \delta_{0}^{-2} \\
& \text { 2) } \frac{\left|f_{0}(0)\right|}{\left|f_{0}^{\prime}(0)\right|}=\frac{\left.|| P\left(x_{0}\right)\right|^{2}}{\left|\left(P\left(x_{0}\right), \quad P^{\prime}\left(x_{0}\right) P\left(x_{0}\right)\right)\right|} \leqslant M_{0} \\
& \text { 3) }\left|f_{0}^{\prime \prime}(\varepsilon)\right|=\left|\left(P\left(x_{0}\right), \quad P^{\prime \prime}\left(x_{0}+\varepsilon P\left(x_{0}\right)\right) P^{2}\left(x_{0}\right)\right)\right| \leqslant B \delta_{0}^{3}
\end{aligned}
$$

для всех $\varepsilon$, удовлетворяющих условию

$$
|\varepsilon| \leqslant \frac{1-\sqrt{1-2 M_{0}^{2} B \bar{\delta}_{0}}}{M_{0}^{2} B \bar{\delta}_{0}} M_{0}
$$

потому что при таких $\varepsilon x_{0}+\varepsilon P\left(x_{0}\right) \in S\left(x_{0}, r\right)$, что видно из неравенства

$$
\left\|\varepsilon P\left(x_{0}\right)\right\| \leqslant \frac{1-\sqrt{1-2 M_{0}^{2} B \bar{\delta}_{0}}}{M_{0}^{2} B \bar{\delta}_{0}} M_{0} \delta_{0}=\frac{1}{B}\left(\frac{1}{M_{0}}-\frac{1}{M_{1}}\right) \frac{\delta_{0}}{\bar{\delta}_{0}}<r
$$

4) $M_{0}^{2} B \bar{\delta}_{0}<1-\frac{M_{0}}{M_{1}}=1-\frac{M_{0} A}{M_{1} A}<1-\frac{1}{\sqrt{2}}<\frac{1}{2}$

так как в силу $2^{\circ}$ и $3^{\circ} M_{0} A \geqslant 1$ и, как мы показали, $M_{1} A<M^{*} A \leqslant \sqrt{2}$.

Таким образом, выполнены все условия теоремы Л. В. Канторовича ([4], стр. 170) о сходимости метода Ньютона для уравнения

$$
f_{0}(\varepsilon)=\left(P\left(x_{0}\right), \quad P\left(x_{0}+\varepsilon P\left(x_{0}\right)\right)\right)=0
$$

где начальное приближение $\varepsilon_{0}^{(0)}=0$. Поэтому уравнение (3) разрешимо при $n=0$, и в силу той же теоремы

$$
\left|\varepsilon_{0}\right| \leqslant \frac{1-\sqrt{1-2 M_{0}^{2} B \bar{\delta}_{0}}}{M_{0}^{2} B \bar{\delta}_{0}} M_{0}=\frac{2 M_{0}}{1+\sqrt{1-2 M_{0}^{2} \overline{B \delta}_{0}}}
$$

где $\varepsilon_{0}-$ наименьшее по абсолютной величине решение уравнения $f_{0}(\varepsilon)=0$. Теперь $\left\|x_{1}-x_{0}\right\|=\left|\varepsilon_{0}\right| \delta_{0}<r$, т. е. $x_{1} \in S\left(x_{0}, r\right)$.

Так как оператор $P(x)$ имеет в сфере $S\left(x_{0}, r\right)$ производную $P^{\prime}(x)$ и $x_{0}, x_{1} \in S\left(x_{0}, r\right)$, то справедлива формула Лагранжа ([5], стр. 55):

$$
\left(P\left(x_{1}\right)-P\left(x_{0}\right), z\right)=\left(P^{\prime}(\bar{x})\left(x_{1}-x_{0}\right), z\right)
$$

где $z-$ произвольный элемент из $H$, а $\bar{x}=x_{0}+\tau\left(x_{1}-x_{0}\right) \in S\left(x_{0}, r\right)$, $0<\tau<1$. Из (12) вытекает, что

$$
\left\|P\left(x_{1}\right)-P\left(x_{0}\right)\right\| \leqslant\left\|P^{\prime}(\bar{x})\left(x_{1}-x_{0}\right)\right\|
$$


Используя (13) и учитывая (7), получим

$\left\|P\left(x_{1}\right)\right\|^{2}+\left\|P\left(x_{0}\right)\right\|^{2}=\left\|P\left(x_{1}\right)-P\left(x_{0}\right)\right\|^{2} \leqslant\left\|P^{\prime}(\bar{x})\left(x_{1}-x_{0}\right)\right\|^{2} \leqslant A^{2} \varepsilon_{0}^{2}\left\|P\left(x_{0}\right)\right\|^{2}$ откуда в силу (11)

$$
\delta_{1} \leqslant \delta_{0} \sqrt{\frac{4 M_{0}^{2} A^{2}}{\left(1+\sqrt{1-2 M_{0}^{2} B \bar{\delta}_{0}}\right)^{2}}-1} \leqslant \bar{\delta}_{0} \sqrt{\frac{4 M_{0}^{2} A^{2}}{\left(1+\sqrt{1-2 M_{0}^{2} B \bar{\delta}_{0}}\right)^{2}}-1}=\bar{\delta}_{1}
$$

Применяя формулу (12) к дифференциалу $P^{\prime}(x) h$, получим $\left|\left(P^{\prime}\left(x_{1}\right) h, h\right)\right|=\left|\left(P^{\prime}\left(x_{0}\right) h, h\right)-\left(P^{\prime}\left(x_{0}\right) h-P^{\prime}\left(x_{1}\right) h, h\right)\right| \geqslant\left|\left(P^{\prime}\left(x_{0}\right) h, h\right)\right|-$

$$
\begin{gathered}
-\left|\left(P^{\prime \prime}(\bar{x}) h\left(x_{0}-x_{1}\right), h\right)\right| \geqslant \frac{1}{M_{0}}\|h\|^{2}-B\left|\varepsilon_{0}\right| \delta_{0}\|h\|^{2} \geqslant \\
\geqslant \frac{1}{M_{0}}\left(1-\frac{1-\sqrt{1-2 M_{0}^{2} B \bar{\delta}_{0}}}{M_{0}^{2} B \bar{\delta}_{0}} M_{0}^{2} B \bar{\delta}_{0}\right)\|h\|^{2}=\frac{1}{M_{1}}\|h\|^{2}
\end{gathered}
$$

где

$$
M_{1}=\frac{M_{0}}{\sqrt{1-2 M_{0}^{2} B \bar{\delta}_{0}}}
$$

Покажем, что $S\left(x_{1}, r_{1}\right) \subset S\left(x_{0}, r\right)$, где $r_{1}=\frac{1}{B}\left(\frac{1}{M_{1}}-\frac{1}{M^{*}}\right) \frac{\delta_{1}}{\delta_{1}}$. Действительно, пусть $x \in S\left(x_{1}, r_{1}\right)$; тогда

$$
\begin{gathered}
\left\|x-x_{0}\right\| \leqslant\left\|x-x_{1}\right\|+\left\|x_{1}-x_{0}\right\| \leqslant \frac{1}{B}\left(\frac{1}{M_{1}}-\frac{1}{M^{*}}\right) \frac{\delta_{1}}{\bar{\delta}_{1}}+ \\
+\frac{1}{B}\left(\frac{1}{M_{0}}-\frac{1}{M_{1}}\right) \frac{\delta_{0}}{\bar{\delta}_{0}} \leqslant \frac{1}{B}\left(\frac{1}{M_{0}}-\frac{1}{M^{*}}\right) \frac{\delta_{0}}{\bar{\delta}_{0}}=r
\end{gathered}
$$

ибо в силу (14) $\frac{\delta_{1}}{\bar{\delta}_{1}} \leqslant \frac{\delta_{0}}{\bar{\delta}_{0}}$. Таким образом, $x \in S\left(x_{0}, r\right)$, т. е. $S\left(x_{1}, r_{1}\right)$ с $\subset S\left(x_{0}, r\right)$. Поэтому оценки, данные условием $3^{\circ}$, сохраняются для всех $x \in S\left(x_{1}, r_{1}\right)$. Так как мы получили также постоянные $\overline{\delta_{1}}, M_{1}$, которые удовлетворяют условиям $1^{\circ}$ и $2^{\circ}$ для $x_{1}$, и $4^{\circ}$ тоже остается в силе, то все жсловия теоремы выполнены для $x_{1}$. Мы можем все рассуждения повторить, заменяя $x_{0}$ везде на $x_{1}$. Таким образом, нетрудно индуктивно доказать, что условия теоремы выполнены для всех $x_{n}$. Попутно получим рекуррентные соотношения (8), (9) и неравенства

$$
\left|\varepsilon_{n}\right| \leqslant \frac{1-\sqrt{1-2 M_{n}^{2} B \bar{\delta}_{n}}}{M_{n}^{2} B \bar{\delta}_{n}} M_{n}
$$

$$
\begin{gathered}
\delta_{n+1} \leqslant \delta_{n} \sqrt{\frac{4 M_{n}^{2} A^{2}}{\left(1+\sqrt{1-2 M_{n}^{2} B \bar{\delta}_{n}}\right)^{2}}-1} \leqslant \bar{\delta}_{n} \sqrt{\frac{4 M_{n}^{2} A^{2}}{\left(1+\sqrt{1-2 M_{n}^{2} B \bar{\delta}_{n}}\right)^{2}}-1}= \\
=\bar{\delta}_{n+1}
\end{gathered}
$$

а также включение $x_{n} \in S\left(x_{0}, r\right)$. 
Покажем, что последовательность $\left\{x_{n}\right\}$ сходится к решению $x^{*}$ уравнения (1), лежащему в сфере $S\left(x_{0}, r\right)$. Для любого $\kappa$ имеем

$$
\begin{gathered}
\left\|x_{k+1}-x_{k}\right\|=\left|\varepsilon_{k}\right| \delta_{k} \leqslant \frac{1-\sqrt{1-2 M_{k}^{2} B \bar{\delta}_{k}}}{M_{k}^{2} B \bar{\delta}_{k}} M_{k} \delta_{k}=\frac{1}{B}\left(\frac{1}{M_{k}}-\frac{1}{M_{k+1}}\right) \frac{\delta_{k}}{\bar{\delta}_{k}}, \\
\frac{\delta_{k+1}}{\bar{\delta}_{k+1}} \leqslant \frac{\delta_{k}}{\bar{\delta}_{k}}
\end{gathered}
$$

Поэтому

$$
\begin{gathered}
\left\|x_{n+p}-x_{n}\right\| \leqslant\left\|x_{n+p}-x_{n+p-1}\right\|+\ldots+\left\|x_{n+2}-x_{n+1}\right\|+\left\|x_{n+1}-x_{n}\right\| \leqslant \\
\leqslant \frac{1}{B}\left(\frac{1}{M_{n+p-1}}-\frac{1}{M_{n+p}}\right) \frac{\delta_{n+p-1}}{\bar{\delta}_{n+p-1}}+\ldots+\frac{1}{B}\left(\frac{1}{M_{n+1}}-\frac{1}{M_{n+2}}\right) \frac{\delta_{n+1}}{\bar{\delta}_{n+1}}+ \\
+\frac{1}{B}\left(\frac{1}{M_{n}}-\frac{1}{M_{n+1}}\right) \frac{\delta_{n}}{\bar{\delta}_{n}} \leqslant \frac{1}{B}\left(\frac{1}{M_{n}}-\frac{1}{M_{n+p}}\right) \frac{\delta_{n}}{\bar{\delta}_{n}}
\end{gathered}
$$

и в силу полноты пространства $H$ существует $x^{*}=\lim x_{n}$. Переход к пределу $(p \rightarrow \infty)$ дает

$$
\left\|x^{*}-x_{n}\right\| \leqslant \frac{1}{B}\left(\frac{1}{M_{n}}-\frac{1}{M^{*}}\right) \frac{\delta_{n}}{\bar{\delta}_{n}}
$$

Из последнего неравенства следует, что $x^{*} \in S\left(x_{0}, r\right)$. Так как в силу $3^{\circ}$ оператор $P(x)$ непрерывен, то

$$
\left\|P\left(x^{*}\right)\right\|=\lim \left\|P\left(x_{n}\right)\right\| \leqslant \lim \bar{\delta}_{n}^{\prime}=0
$$

откуда следует, что $x^{*}$ - решение уравнения (1).

Для всех $\bar{x} \in S\left(x_{0}, r\right)$

$$
\left.\mid\left(P^{\prime} \bar{x}\right) h, h\right) \mid \geqslant \frac{1}{M_{0}}\left(1-M_{0} B\left\|\bar{x}-x_{0}\right\|\right)\|h\|^{2} \geqslant \frac{1}{M_{0}}\left(1-M_{0} B r\right)\|h\|^{2}
$$

Так как $x^{*} \in S\left(x_{0}, r\right) \quad$ и $x_{n} \in S\left(x_{0}, r\right)$, то, пользуясь формулой $(12)$, получим

$$
\begin{gathered}
\left\|P\left(x_{n}\right)\right\|\left\|x_{n}-x^{*}\right\| \geqslant\left|\left(P\left(x_{n}\right)-P\left(x^{*}\right), x_{n}-x^{*}\right)\right|= \\
=\left|\left(P^{\prime}(\bar{x})\left(x_{n}-x^{*}\right), x_{n}-x^{*}\right)\right| \geqslant \frac{1}{M_{0}}\left(1-M_{0} B r\right)\left\|x_{n}-x^{*}\right\|^{2}
\end{gathered}
$$

откуда

$$
\left\|x^{*}-x_{n}\right\| \leqslant \frac{M_{0}}{1-M_{0} B r} \delta_{n} \leqslant M^{*} \delta_{n} \leqslant \frac{V \overline{2}}{A} \delta_{n}
$$

Теорема полностью доказана.

3. Рассмотрим вопрос о сходимости последовательностей $\left\{M_{n}\right\}$, $\left\{\bar{\delta}_{n}\right\}$. Введем обозначения

$$
a_{n}=2 M_{n}^{2} \mathrm{~B} \bar{\delta}_{n}, \quad b_{n}=M_{n}^{2} A^{2}
$$

Тогда, в силу (8) и (9), получим соотношения 


$$
\begin{aligned}
& a_{n+1}=\frac{a_{n}}{1-a_{n}} \sqrt{\frac{4 b_{n}}{\left(1+\sqrt{1-a_{n}}\right)^{2}}-1} \\
& b_{n+1}=\frac{b_{n}}{1-a_{n}}
\end{aligned}
$$

Имеет место следующая

Те ор ема 2. Если $a_{0}<1$ и существует такое вещественное число $k>0$, что $\alpha=k^{2} a_{0}<2$, а также выполнены неравенства:

$$
\begin{aligned}
& 1^{\circ} 4 b_{0} \leqslant\left(2-a_{0}\right)^{2}\left[1+\left(\frac{k}{k+1}\right)^{4}\left(1-a_{0}\right)^{2}\right] \\
& 2^{\circ}\left(2-a_{0}\right)^{2}\left[1+\left(\frac{k}{k+1}\right)^{4}\left(1-a_{0}\right)^{2}\right] \leqslant \\
& \leqslant\left(1-a_{0}\right)\left(2-\frac{k^{2} a_{0}}{(k+1)^{2}}\right)^{2}\left[1+\left(\frac{k+1}{k+2}\right)^{4}\left(1-\frac{k^{2} a_{0}}{(k+1)^{2}}\right)^{2}\right]
\end{aligned}
$$

то последовательности $\left\{a_{n}\right\},\left\{b_{n}\right\}$ (как $\left.u\left\{M_{n}\right\} u\left\{\bar{\delta}_{n}\right\}\right)$ сходятся. Пра этом

$$
\left.\begin{array}{c}
a_{n}<\left(\frac{k}{k+n}\right)^{2} a_{0} \\
b_{n}<\frac{1}{4}\left(2-\frac{\mathrm{k}^{2} a_{0}}{(k+n)^{2}}\right)^{2}\left[1+\left(\frac{k+n}{k+n+1}\right)^{4}\left(1-\frac{k^{2} u_{0}}{(k+n)^{2}}\right)^{2}\right]
\end{array}\right\}
$$

Док аз а тельст в о. Обозначим

$$
\begin{gathered}
F(u, \alpha)=\left(1-\frac{a}{u^{2}}\right)\left(2-\frac{a}{(u+1)^{2}}\right)^{2}\left[1+\left(\frac{u+1}{u+2}\right)^{4}\left(1-\frac{a}{(u+1)^{2}}\right)^{2}\right]- \\
-\left(2-\frac{a}{u^{2}}\right)^{2}\left[1+\left(\frac{u}{u+1}\right)^{4}\left(1-\frac{a}{u^{2}}\right)^{2}\right]
\end{gathered}
$$

где $\alpha=k^{2} a_{0}$. Теперь условие $2^{\circ}$ можно записать в виде

$$
F(k, \alpha) \geqslant 0
$$

Покажем, что из этого неравенства следуют неравенства

$$
F(k+n, \alpha)>0 \quad n=1,2,3, \ldots
$$

Для доказательства воспользуемся методом математической индукции. Достаточно показать, что из неравенства $F(u, \alpha) \geqslant 0$, где $u \geqslant k>0$, $0<\alpha<2$, следует неравенство $F(u+1, \alpha)>0$. Для того, чтобы это доказать, приведем в выражении $F(u, \alpha)$ все члены к общему знаменателю

где

$$
F(u, \alpha)=\frac{f(u, \alpha)}{\varphi(u)}
$$

$$
\begin{aligned}
f(u, \alpha)=\{ & \left.(u+2)^{4}+\left[(u+1)^{2}-\alpha\right]^{2}\right\}\left[2(u+1)^{2}-\alpha\right]^{2}\left(u^{2}-\alpha\right) u^{2}- \\
& -\left\{(u+1)^{4}+\left(u^{2}-\alpha\right)^{2}\right\}\left(2 u^{2}-\alpha\right)^{2}(u+2)^{4}
\end{aligned}
$$

и

$$
\varphi(u)=[u(u+1)(u+2)]^{4}>0
$$

Таким образом, $F(u, \alpha)$ и $f(u, \alpha)$ имеют один и тот же знак, и поэтому 
достаточно показать, что из неравенства $f(u, \alpha) \geqslant 0$ следует неравенство $f(u+1, \alpha)>0$.

После разложения $f(u, \alpha)$ и $f(u+1, \alpha)$ по степеням $u+1$ можно непосредственно проверить, что выражение

$$
\begin{aligned}
\psi(u, \alpha)= & f(u+1, \alpha)-\left[1+\frac{10}{u+1}+\frac{49}{(u+1)^{2}}+\frac{156+4 a}{(u+1)^{3}}+\right. \\
& \left.+\frac{330+31 a}{(u+1)^{4}}+\frac{334+90 a+a^{2}}{(u+1)^{5}}\right] f(u, \alpha)
\end{aligned}
$$

положительно при всех $u>0,0<\alpha<2$. Отсюда следует, что $f(u+1, \alpha)>0$, а также $F(u+1, \alpha)>0$. Но тогда выполнено и $(20)$.

В силу условия $1^{\circ}$ (и учитывая, что $a_{0}<1$ ) имеем

$$
\frac{4 b_{0}}{\left(1+\sqrt{1-a_{0}}\right)^{2}}-1<\frac{4 b_{0}}{\left(2-a_{0}\right)^{2}}-1 \leqslant\left(\frac{k}{k+1}\right)^{4}\left(1-a_{0}\right)^{2}
$$

В силу (17) получим отсюда

$$
a_{1}<\left(\frac{k}{k+1}\right)^{2} a_{0}
$$

Учитывая (18), получим, в силу $1^{\circ}$ и $2^{\circ}$

$$
\begin{aligned}
& 4 b_{1}=\frac{4 b_{0}}{1-a_{0}} \leqslant \frac{\left(2-a_{0}\right)^{2}}{1-a_{0}}\left[1+\left(\frac{k}{k+1}\right)^{4}\left(1-a_{0}\right)^{2}\right] \leqslant \\
& \leqslant\left(2-\frac{k^{2} a_{0}}{(k+1)^{2}}\right)^{2}\left[1+\left(\frac{k+1}{k+2}\right)^{4}\left(1-\frac{k^{2} a_{0}}{(k+1)^{2}}\right)^{2}\right]
\end{aligned}
$$

Повторяя таким образом все рассуждения и учитывая при этом неравенство $(21)$, а вместо $1^{\circ}$ и $2^{\circ}$ соответственно $(22)$ и $(20)$, получим неравенства (19) для $n=2$. При помощи индукции получим неравенства (19) для всех $n$. Из них вытекает сходимость последовательностей $\left\{a_{n}\right\}$ и $\left\{b_{n}\right\}$; вместе с тем получим:

или

$$
\lim a_{n}=0, \quad \lim b_{n} \leqslant 2
$$

$$
\lim \bar{\delta}_{n}=0, \quad \lim M_{n} \leqslant \frac{\sqrt{2}}{A}
$$

4. Рассмотрим теперь одну модификацию метода наискорейшего спуска, практическое применение которой значительно проще, но точность почти та же самая, что и у метода $\{(2),(3)\}$. Постоянные $\varepsilon_{n}$ находим приближенно следующим образом. Уравнение (3), которое может быть записано в виде

$$
\left(P\left(x_{n}\right), P\left(x_{n}\right)+P^{\prime}\left(x_{n}\right) \varepsilon P\left(x_{n}\right)+R\left(x_{n}, \varepsilon P\left(x_{n}\right)\right)\right)=0
$$

где

$$
R\left(x_{n}, \varepsilon P\left(x_{n}\right)\right)=\int_{0}^{1}(1-\lambda) P^{\prime \prime}\left(x_{n}+\lambda \varepsilon P\left(x_{n}\right)\right) \varepsilon^{2} P^{2}\left(x_{n}\right) d \lambda,
$$

заменим приближенным

$$
\left(P\left(x_{n}\right), P\left(x_{n}\right)+P^{\prime}\left(x_{n}\right) \varepsilon P\left(x_{n}\right)\right)=0
$$

Решение $\varepsilon_{n}$ уравнения (24) выражается просто: 


$$
\varepsilon_{n}=-\frac{\left\|P\left(x_{n}\right)\right\|^{2}}{\left(P\left(x_{n}\right), P^{\prime}\left(x_{n}\right) P\left(x_{n}\right)\right)}
$$

Отсюда получим следующую рекуррентную формулу для нахождения приближений уравнения (1):

$$
x_{n+1}=x_{n}-\frac{\left\|P\left(x_{n}\right)\right\|^{2}}{\left(P\left(x_{n}\right), P^{\prime}\left(x_{n}\right) P\left(x_{n}\right)\right)} P\left(x_{n}\right)
$$

Этот метод рассмотрен и в статьях $\left.{ }^{2}\right]$ и $\left[{ }^{3}\right]$.

Отметим, что если в (1) $P(x)$ - вещественная функция вещественной переменной, то метод (26) дает метод Ньютона, рассмотренный B [ $\left.{ }^{4}\right]$.

Справедлива следующая теорема:

Те орем а 3. Пусть выполнены условия:

$1^{\circ}\left\|P\left(x_{0}\right)\right\|=\delta_{0} \leqslant \bar{\delta}_{0} \quad\left(x_{0}-\right.$ начальное приближение $)$;

$2^{\circ}\left|\left(P^{\prime}\left(x_{0}\right) h, h\right)\right| \geqslant \frac{1}{M_{0}}\|h\|^{2}, \quad M_{0}>0, \quad h \in H ;$

$3^{\circ}$ для всех $x \in S\left(x_{0}, r\right)$, где $r=\frac{1}{B}\left(\frac{1}{M_{0}}-\frac{1}{M^{*}}\right) \frac{\delta_{0}}{\bar{\delta}_{0}}$, оператор $P(x)$ дважды дифференцируем, и справедливы оценки

$$
\left\|P^{\prime}(x)\right\| \leqslant A, \quad\left\|P^{\prime \prime}(x)\right\| \leqslant B ;
$$

$4^{\circ}$ величины $M_{0}, \bar{\delta}_{0}$, А и В такие, что последовательности $\left\{M_{n}\right\},\left\{\bar{\delta}_{n}\right\}$, вычисленные из рекуррентных соотночений

$$
\begin{gathered}
M_{n+1}=\frac{M_{n}}{1-M_{n}^{2} B \bar{\delta}_{n}}, \quad n=0,1,2, \ldots \\
\bar{\delta}_{n+1}=\bar{\delta}_{n} \sqrt{M_{n}^{2} A^{2}+M_{n}^{2} B \bar{\delta}_{n}-1}, \quad n=0,1,2, \ldots
\end{gathered}
$$

сходятся (так что $M^{2}{ }_{n} B \bar{\delta}_{n}<1$ для всех $\left.n\right)$.

Тогда уравнение (1) имеет в сфере $S\left(x_{0}, r\right)$ решение $x^{*}, \kappa$ которому сходится полученная из (26) последовательность $\left\{x_{n}\right\}, u$ справедлива оценка

$$
\left\|x^{*}-x_{n}\right\| \leqslant M^{*} \delta_{n} \leqslant \frac{V \overline{2}}{A} \delta_{n}
$$

где $M^{*}=\lim M_{n}, \delta_{n}=\left\|P\left(x_{n}\right)\right\|$.

Доказ ат ельство аналогично доказательству теоремы 1. Как и раньше, получим, что $M_{0} A \geqslant 1$ и $M_{n+1}>M_{n}$. Из требования сходимости последовательностей $(27)$ и $(28)$ следует, что $\lim \bar{\delta}_{n}=0$ и $M^{*}=\lim M_{n} \leqslant$ $\leqslant \frac{\bar{v} \bar{s}}{A}$.

Пользуясь оценками $1^{\circ}, 2^{\circ}$ и соотношением (27), получим

$$
\left\|x_{1}-x_{0}\right\| \leqslant M_{0} \delta_{0}=\frac{1}{B}\left(\frac{1}{M_{0}}-\frac{1}{M_{1}}\right) \frac{\delta_{0}}{\bar{\delta}_{0}}<\frac{1}{B}\left(\frac{1}{M_{0}}-\frac{1}{M^{*}}\right) \frac{\delta_{0}}{\bar{\delta}_{0}}=r
$$

т. е. $x_{1} \in S\left(x_{0}, r\right)$. В силу соотношений (23) и (25)

$$
\left(P\left(x_{n+1}\right), P\left(x_{n}\right)\right)=\left(P\left(x_{n}\right), R\left(x_{n}, \varepsilon_{n} P\left(x_{n}\right)\right)\right)
$$


Так как справедлива оценка

$$
\left\|R\left(x_{n}, \varepsilon_{n} P\left(x_{n}\right)\right)\right\| \leqslant \frac{1}{2} \varepsilon_{n}^{2}\left\|P\left(x_{n}\right)\right\|_{0 \leqslant \lambda \leqslant 1}^{2} \max _{0 \leqslant 1}\left\|P^{\prime \prime}\left(x_{n}+\lambda \varepsilon_{n} P\left(x_{n}\right)\right)\right\|
$$

$x_{0}+\lambda \varepsilon_{0} P\left(x_{0}\right) \in S\left(x_{0}, r\right)$ для всех $\lambda \in[0,1]$, а в силу $2^{\circ}$ и $(25)\left|\varepsilon_{0}\right| \leqslant M_{0}$, то имеем

$$
\left|\left(P\left(x_{1}\right), P\left(x_{0}\right)\right)\right| \leqslant \frac{1}{2} M_{0}^{2} B \bar{\delta}_{0}\left\|P\left(x_{0}\right)\right\|^{2}
$$

Теперь

$$
\begin{gathered}
\left\|P\left(x_{1}\right)\right\|^{2}-2\left(P\left(x_{1}\right), P\left(x_{0}\right)\right)+\left\|P\left(x_{0}\right)\right\|^{2}=\left\|P\left(x_{1}\right)-P\left(x_{0}\right)\right\|^{2} \leqslant \\
\leqslant\left\|P^{\prime}(\bar{x})\left(x_{1}-x_{0}\right)\right\|^{2} \leqslant A^{2} M_{0}^{2}\left\|P\left(x_{0}\right)\right\|^{2}
\end{gathered}
$$

откуда

$$
\delta_{1}^{2} \leqslant\left(M_{0}^{2} A^{2}+M_{0}^{2} B \bar{\delta}_{0}-1\right) \delta_{0}^{2} \leqslant\left(M_{0}^{2} A^{2}+M_{0}^{2} B \bar{\delta}_{0}-1\right) \bar{\delta}_{0}^{2}=\bar{\delta}_{1}^{2}
$$

Как и в доказательстве теоремы 1 , получим

$$
\left|\left(P^{\prime}\left(x_{1}\right) h, h\right)\right| \geqslant \frac{1}{M_{1}}\|h\|^{2}, \quad h \in H
$$

где

$$
M_{1}=\frac{M_{0}}{1-M_{0}^{2} B \bar{\delta}_{0}}
$$

И здесь $S\left(x_{1}, r_{1}\right) \subset S\left(x_{0}, r\right)$, где $r_{1}=\frac{1}{B}\left(\frac{1}{M_{1}}-\frac{1}{M^{*}}\right) \frac{\delta_{1}}{\bar{\delta}_{1}}$, поэтому оценки для $\left\|P^{\prime}(x)\right\|$ и $\left\|P^{\prime \prime}(x)\right\|$ остаются в силе при переходе от $x_{0}$ к $x_{1}$.

Окончания доказательств теорем 3 и 1 полностью совпадают, и мы не будем здесь их повторять.

5. Обозначим

$$
a_{n}=M_{n}^{2} B \bar{\delta}_{n}, \quad b_{n}=M_{n}^{2} A^{2}
$$

Тогда в силу (27) и (28) получим соотношения

$$
\begin{aligned}
& a_{n+1}=\frac{\sqrt{b_{n}+a_{n}-1}}{\left(1-a_{n}\right)^{2}} a_{n} \\
& b_{n+1}=\frac{b_{n}}{\left(1-a_{n}\right)^{2}}
\end{aligned}
$$

О сходимости последовательностей $\left\{a_{n}\right\},\left\{b_{n}\right\}$ имеем место

Те орем а 4 . Если $a_{0}<1$ и существует такое вещественное число $k>0$, что $\alpha=k^{2} a_{0}<1$, а также выполнены неравенства:

$$
\begin{aligned}
& 1^{\circ} b_{0}+a_{0}-1 \leqslant\left(\frac{k}{k+1}\right)^{4}\left(1-a_{0}\right)^{4}, \\
& 2^{\circ} \frac{k^{4}}{\left(k^{2}-a\right)^{2}}\left[\frac{k^{2}-a}{k^{2}}+\left(\frac{k^{2}-a}{k(k+1)}\right)^{4}\right] \leqslant \frac{(k+1)^{2}-a}{(k+1)^{2}}+\left(\frac{(k+1)^{2}-a}{(k+1)(k+2)}\right)^{4},
\end{aligned}
$$

то последовательности $\left\{a_{n}\right\},\left\{b_{n}\right\}$, вычисленные из (31), (32) (каки иоследовательности $\left\{M_{n}\right\},\left\{\bar{\delta}_{n}\right\}$, вычисленные из (27), (28)), сходятся.

Доказательство аналогично доказательству теоремы 2. 
6. Если в теоремах 1 и 3 условие $2^{\circ}$ заменить более строгим условием (ср. $\left[{ }^{2}\right],\left[{ }^{3}\right]$ и $\left.\left[{ }^{1}\right]\right)$

$$
\left|\left(P^{\prime}(x) h, h\right)\right| \geqslant \frac{1}{M}\|h\|^{2}
$$

то имеют место теоремы 5 и 6.

Теорем а 5. Пусть выполнены условия:

$1^{\circ}$ для всех $x \in S\left(x_{0}, r\right)$, где $r=\frac{M \delta_{0}}{1-q}$, оператор $P(x)$ дважды дифференцируем и

a) $\left\|P^{\prime}(x)\right\| \leqslant A$, б) $\left\|P^{\prime \prime}(x)\right\| \leqslant B$,

в) $\left|\left(P^{\prime}(x) h, h\right)\right| \geqslant \frac{1}{M}\|h\|^{2}, \quad M>0, \quad h \in H$;

$2^{\circ} q=\sqrt{M^{2} A^{2}-1}<1$;

$3^{\circ} M^{2} B \delta_{0} \leqslant \frac{1}{2}, 1-\sqrt{1-2 M^{2} B \delta_{0}}<2 \sqrt{M^{2} A^{2}-1}$.

Тогда уравнение (1) имеет в сфере $S\left(x_{0}, r\right)$ решение $x^{*}$, к которому сходится последовательность $\left\{x_{n}\right\}$, полученная методом $\{(2),(3)\}$. Имеет место оценка

$$
\left\|x^{*}-x_{n}\right\| \leqslant M \delta_{n} \leqslant M \delta_{0} q^{n}
$$

где $\delta_{n} \geqslant\left\|P\left(x_{n}\right)\right\| \quad(n=0,1,2, \ldots)$.

Эту теорему в несколько ином виде, без требования дифференцируемости оператора, дал Ю. Лумисте [']. Здесь уточнены область $S\left(x_{0}, r\right)$ и оценка погрешности. Эти уточнения легко получить, если иметь в виду доказательство теоремы 1.

Т е о рем а 6. Пусть выполнены условия:

$1^{\circ}$ для всех $x \in S\left(x_{0}, r\right)$, где $r=\frac{M \delta_{0}}{1-q}$, оператор $P(x)$ дважды дифференцируем $и$
a) $\left\|P^{\prime}(x)\right\| \leqslant A$,
б) $\left\|P^{\prime \prime}(x)\right\| \leqslant B$,
в) $\left|\left(P^{\prime}(x) h, h\right)\right| \geqslant \frac{1}{M}\|h\|^{2}, \quad M>0, \quad h \in H$;

$2^{\circ} q=\sqrt{M^{2} A^{2}+M^{2} B \delta_{0}-1}<1$.

Тогда уравнение (1) имеет в сфере $S\left(x_{0}, r\right)$ решение $x^{*}, \kappa$ которому сходится последовательность $\left\{x_{n}\right\}$, полученная методом (26). Нмеет место оценка

где $\delta_{n} \geqslant\left\|P\left(x_{n}\right)\right\| \quad(n=0,1,2, \ldots)$.

$$
\left\|x^{*}-x_{n}\right\| \leqslant M \delta_{n} \leqslant M \delta_{0} q^{n}
$$

7. Рассмотрим вопрос о единственности решения уравнения

Покажем, что имеет место

Те орем а 7 . Пусть

$1^{\circ}\left|\left(P^{\prime}\left(x_{0}\right) h, h\right)\right| \geqslant \frac{1}{M_{0}}\|h\|^{2}, M_{0}>0, h \in H ;$ 


\section{$2^{\circ}$ в cqepe $S\left(x_{0}, r\right)$}

$$
\left\|P^{\prime \prime}(x)\right\| \leqslant B
$$

$3^{\circ} \mathrm{M}_{0} \mathrm{Br}<1$.

Тогда уравнение (1) имеет в сфере $S\left(x_{0}, r\right)$ не более одного решения.

Доказ а тельство. Пусть $x^{*} \in S\left(x_{0}, r\right)$ есть решение уравнения (1). Допустим, что уравнение (1) имеет в этой сфере еще другое решение $x^{* *}$. Пользуясь условием $1^{\circ}$, получим, как в доказательстве теоремы 1 ,

$$
\left|\left(P^{\prime}(\bar{x}) h, h\right)\right| \geqslant \frac{1}{M_{0}}\left(1-M_{0} B r\right)\|h\|^{2}
$$

если $\bar{x} \in S\left(x_{0}, r\right)$. Возьмем $h=x^{*}-x^{* *}$. Тогда в силу

$$
\begin{gathered}
0=\left|\left(P\left(x^{*}\right)-P\left(x^{* *}\right), x^{*}-x^{* *}\right)\right|= \\
=\left|\left(P^{\prime}(\bar{x})\left(x^{*}-x^{* *}\right), x^{*}-x^{* *}\right)\right| \geqslant \frac{1}{M_{0}}\left(1-M_{0} B r\right)\left\|x^{*}-x^{* *}\right\|^{2}
\end{gathered}
$$

где $\bar{x}=x^{* *}+\tau\left(x^{*}-x^{* *}\right) \in S\left(x_{0}, r\right), 0<\tau<1$. Так как $M_{0} B r<1$, то отсюда следует, что

$$
x^{*}=x^{* *}
$$

Теорема доказана.

Еще легче доказать следующую теорему:

Т еор ем а 8 . Если для всех $x \in S\left(x_{0}, r\right)$

$$
\left|\left(P^{\prime}(x) h, h\right)\right| \geqslant \frac{1}{M}\|h\|^{2}, \quad 0<M<\infty, h \in H
$$

то уравнение (1) имеет в сфере $S\left(x_{0}, r\right)$ не более одного решения.

След стви е. Если существует такая постоянная $M, 0<M<\infty$, что условие (34) выполнено для всех $x \in H$, то уравнение (1) не может иметь в $H$ более одного решения.

8. В качестве п р и м е а рассмотрим интегральное уравнение

$$
P(x)=x(s)-\frac{1}{5} \int_{0}^{\frac{1}{2}} \sqrt{s+t} \cos x(t) d t=0
$$

к которому применяем метод (26). Возьмем за начальное приближение $x_{0}(s)=0$. В качестве пространства $H$ выберем множество функций $\boldsymbol{L}^{2}\left(0, \frac{1}{2}\right)$ с интегрируемым квадратом. В рассматриваемом случае легко получить следующие оценки:

$$
\begin{gathered}
\left\|P^{\prime}(x)\right\| \leqslant 1+0,05 \sqrt{2}<1,071=A \\
\left\|P^{\prime \prime}(x)\right\| \leqslant 0,05 \sqrt{2}<0,07072=B
\end{gathered}
$$

Так как $\left(P^{\prime}\left(x_{0}\right) h, h\right)=\|h\|^{2}$, то можем взять $M_{0}=1$. Вычисление $\left\|P\left(x_{0}\right)\right\|$ дает

Tenерь

$$
\left\|P\left(x_{0}\right)\right\|<0,049372<0,04938=\bar{\delta}_{0}
$$

$$
a_{0}=M_{0}^{2} B \bar{\delta}_{0}=0,003421, \quad b_{0}=M_{0}^{2} A^{2}=1,147
$$


Если выбрать $k=2$, то условия теоремы 4 выполнены. Поэтому последовательности $\left\{M_{n}\right\}$ и $\left\{\bar{\delta}_{n}\right\}$, полученные из (27) и (28), сходятся. Тогда выполнены и условия теоремы 3 , откуда следует, что последовательность приближений $\left\{x_{n}\right\}$, вычисленная методом (26), сходится к решению уравнения (35).

Применение метода (26) дает следующие результаты:

$$
\begin{aligned}
\varepsilon_{0} & =-1 \\
x_{1}(s) & =-P\left(x_{0}(s)\right)=\frac{2}{15}\left(\sqrt{\left(s+\frac{1}{2}\right)^{3}}-\sqrt{s^{3}}\right) \\
\varepsilon_{1} & =-0,9949385
\end{aligned}
$$

$x_{2}(s)=0,00067487\left(\sqrt{\left(s+\frac{1}{2}\right)^{3}}-\sqrt{s^{3}}\right)+0,01177164 \sqrt{s+0,02345504}+$ $+0,02376868 \sqrt{s+0,11538267}+0,02823129 \sqrt{s+0,25000000}+$ $+0,02373579 \sqrt{s+0,38461733}+0,01174402 \sqrt{s+0,47654496}$.

Формула (29) дает следующие оценки погрешностей:

$$
\begin{aligned}
& \left\|x^{*}-x_{0}\right\|<6,6 \cdot 10^{-2} \\
& \left\|x^{*}-x_{1}\right\|<1,7 \cdot 10^{-4} \\
& \left\|x^{*}-x_{2}\right\|<2 \cdot 10^{-8}
\end{aligned}
$$

Здесь при вычислении $\varepsilon_{1}, x_{2}(s)$ и оценок погрешностей для $x_{1}$ и $x_{2}$ использована квадратурная формула Гаусса для случая пяти ординат. Погрешность, возникшая из-за пользования квадратурной формулой, не принята в расчет. Впрочем, она незначительна.

В рассматриваемом случае применима и теорема 6 , так как (даже независимо от $x$ )

$$
\left|\left(P^{\prime}(x) h, h\right)\right| \geqslant(1-0,05 \sqrt{2})\|h\|^{2}
$$

и мы можем взять $M=\frac{1}{1-0,05 \sqrt{2}}$. Теперь

$$
q=\sqrt{M^{2} A^{2}+M^{2} B \delta_{0}-1}<0,577<1
$$

значит, условия теоремы 6 выполнены. Апостериорная оценка $\left\|x^{*}-x_{n}\right\| \leqslant M \delta_{n}$ теоремы 6 дает практически те же самые результаты, что и формула (29), но априорная оценка $\left\|x^{*}-x_{n}\right\| \leqslant M \delta_{0} q^{n}$ значительно грубее.

Теорема 7 гарантирует, что решение уравнения (35) единственно в каждой сфере $S(0, r)$, радиус $r$ которой меньше $10 \sqrt{2}$. Но, так как найдется такая постоянная $M=\frac{1}{1-0,05 \sqrt{2}}$, для которой условие (34) теоремы 8 выполнено независимо от $x$, то уравнение (35) имеет во всем множестве $L^{2}\left(0, \frac{1}{2}\right)$ единственное решение. 


\section{ЛИТЕ Р А Т У Р А}

1. Ю. Л у м и т е, Метод наискорейшего спуска при нелинейных уравнениях, Уч. зап. Тартуского гос. ун-та, № $37,1955,106-111$.

2. Гу ан ц Чж а -ч жи, О методе наискорейшего спуска для решения нелинейных операторных уравнений, Шусюэ сюэбао (Acta math. sinica), 6, № 4, 1956, $638-650$.

3. M. Alt m a n, Connection between the method of steepest descent and Newton's method, Bull. Acad. Polon. Sci., Cl. III, 5, No. 11, 1957, 1031-1036.

4. Л. В. К а н то р о в и ч, Функциональный анализ и прикладная математика, Успехи матем. наук, 3, вып. 6, 1948, 89-185.

5. М. М. В а й н бе р г, Вариационные методы исследования нелинейных операторов, M., 1956.

Ннститут энергетики

Академии наук Эстонской ССР
Поступила в редакцию

2. VI 1959

\section{KIIREIMA LANGUSE MEETODIST MITTELINEAARSETE VORRANDITE LAHENDAMISEKS}

\section{Kivistik}

\section{Resümee}

Olgu $P(x)$ mittelineaarne, Fréchet' mõttes kaks korda, diferentseeruv operaator Hilberti ruumist $H$ samasse ruumi. Artiklis käsitletakse võrrandi

$$
P(x)=0
$$

ligikaudset lahendamist kiireima languse meetodil, mille rakendamine seisneb võrrandi lähislahendite leidmises rekurrentsest seosest

$$
x_{n+1}=x_{n}+\varepsilon_{n} P\left(x_{n}\right) \quad(n=0,1,2 \ldots)
$$

kus $x_{0}$ on antud alglähend ja $\varepsilon_{n}$ vōrrandi

$$
\left(P\left(x_{n}\right), P\left(x_{n}+\varepsilon P\left(x_{n}\right)\right)\right)=0
$$

vähima absoluutväärtusega lahend. Tõestatakse t e o r e e m:

Kui

$1^{\circ} \quad\left\|P\left(x_{0}\right)\right\|=\delta_{0} \leqslant \bar{\delta}_{0}$;

$2^{\circ}\left|\left(P^{\prime}\left(x_{0}\right) h, h\right)\right| \geqslant \frac{1}{M_{0}}\|h\|^{2}, \quad M_{0}>0, \quad h \in H$;

$3^{\circ}\left\|P^{\prime}(x)\right\| \leqslant A,\left\|P^{\prime \prime}(x)\right\| \leqslant B$ iga $x$ puhul kinnisest sfäärist $S\left(x_{0}, r\right)$, kus

$$
r=\frac{1}{B}\left(\frac{1}{M_{0}}-\frac{1}{M^{*}}\right) \frac{\delta_{0}}{\bar{\delta}_{0}}
$$

$4^{\circ}$ konstandid $M_{0}, \bar{\delta}_{0}, A, B$ on sellised, et koonduvad rekurrentsetest seostest

$$
M_{n+1}=\frac{M_{n}}{\left.\sqrt{1-2 M_{n}^{2} B \bar{\delta}_{n}}\right)}, \quad \bar{\delta}_{n+1}=\bar{\delta}_{n} \sqrt{\frac{4 M_{n}^{2} A^{2}}{\left(1+\sqrt{\left.1-2 M_{n}^{2} B \bar{\delta}_{n}\right)^{2}}\right.}-1}
$$

arvutatud jadad $\left\{M_{n}\right\}$ ja $\left\{\bar{\delta}_{n}\right\}$;

siis võrrandil (1) on sfääris $S\left(x_{0}, r\right)$ lahend $x^{*}$, milleks koondub seosest (2) arvulatud lähislahendite jada $\left\{x_{n}\right\}$ kiirusega

$$
\left\|x^{*}-x_{n}\right\| \leqslant M^{*} \delta_{n} \leqslant \frac{\sqrt{2}}{A} \delta_{n}
$$

kus $M^{*}=\lim M_{n}$ ja $\delta_{n}=\left\|P\left(x_{n}\right)\right\|$. 

järgi

Edasi vaadeldakse meetodi üht modifikatsiooni, mille puhul $\varepsilon_{n}$ arvutatakse valemi

$$
\varepsilon_{n}=-\frac{\left\|P\left(x_{n}\right)\right\|^{2}}{\left(P^{\prime}\left(x_{n}\right) P\left(x_{n}\right), P\left(x_{n}\right)\right)}
$$
näide.

Artikli lõpuosas tuuakse kaks teoreemi lahendi ainsuse kohta ja lahendatakse üks

Eesti NSV Teaduste Akadeemia

Energeetika Instituut
Saabus toimetusse

2. VI 1959

\section{ON THE METHOD OF STEEPEST DESCENT FOR SOLVING NON-LINEAR EQUATIONS}

\section{Kivistik}

\section{Summary}

Let $H$ be a real Hilbert space and let $P(x)$ be a non-linear operator from $H$ into $H$ which is twice differentiable in the sense of Fréchet. The present paper considers the method of the steepest descent for solving the equation

$$
P(x)=0
$$

The method may be defined by the following formula:

$$
x_{n+1}=x_{n}+\varepsilon_{n} P\left(x_{n}\right) \quad(\mathrm{n}=0,1,2, \ldots)
$$

where $x_{0}$ is the initial approximate solution and $\varepsilon_{n}$ is the solution (which has the smallest absolute value) of the equation

$$
\left(P\left(x_{n}\right), P\left(x_{n}+\varepsilon P\left(x_{n}\right)\right)\right)=0
$$

The following theorem is proved:

If

$$
\begin{aligned}
& 1^{\circ} \quad\left\|P\left(x_{0}\right)\right\|=\delta_{0} \leqslant \bar{\delta}_{0} ; \\
& 2^{\circ} \quad\left|\left(P^{\prime}\left(x_{0}\right) h, h\right)\right| \geqslant \frac{1}{M_{0}}\|h\|^{2}, \quad M_{0}>0, \quad h \in H ;
\end{aligned}
$$

$3^{\circ}\left\|P^{\prime}(x)\right\| \leqslant A,\left\|P^{\prime \prime}(x)\right\| \leqslant B$ for every $x \in S\left(x_{0}, r\right)$, where $S\left(x_{0}, r\right)$ is the closed sphere with the radius $r=\frac{1}{B}\left(\frac{1}{M_{0}}-\frac{1}{M^{*}}\right) \frac{\delta_{0}}{\bar{\delta}_{0}}$

$4^{\circ}$ the constants $M_{0}, \bar{\delta}_{0}, A$ and $B$ are such that the sequences $\left\{M_{n}\right\}$ and $\left\{\bar{\delta}_{n}\right\}$, defined by the recurrence formulae

$$
M_{n+1}=\frac{M_{n}}{\sqrt{1-2 M_{n}^{2} B \bar{\delta}_{n}}}, \quad \bar{\delta}_{n+1}=\bar{\delta}_{n} \sqrt{\frac{4 M_{n}^{2} A^{2}}{\left(1+\sqrt{1-2 M_{n}^{2} B \bar{\delta}_{n}}\right)^{2}}-1}
$$

converge;

then equation (1) has a solution $x^{*}$ in the sphere $S\left(x_{0}, r\right)$ and the sequence of approximate solutions $x_{n}$, defined by $\{(2),(3)\}$, converges to $x^{*}$. For the error estimate we have the formula 


$$
\left\|x^{*}-x_{n}\right\| \leqslant M^{*} \delta_{n} \leqslant \frac{\sqrt{2}}{A} \delta_{n}
$$

where $M^{*}=\lim M_{n}$ and $\delta_{n}=\left\|P\left(x_{n}\right)\right\|$.

A modification of the method of steepest descent is also considered. In this case $\varepsilon_{n}$ is defined by the formula

$$
\varepsilon_{n}=-\frac{\left\|P\left(x_{n}\right)\right\|^{2}}{\left(P^{\prime}\left(x_{n}\right) P\left(x_{n}\right), P\left(x_{n}\right)\right)}
$$

At the end of the paper two theorems are given on the uniqueness of the solution, and an example is solved.

Academy of Sciences of the Estonian S.S.R.,

Received

Institute of Energetics

June 2nd, 1959 